\title{
Creation of novel training programmes mapped to the Joint Royal Colleges of Physicians Training Board Internal Medicine stage 1 curriculum
}

\author{
Authors: Marie McNulty, ${ }^{A}$ Elizabeth Carty, ${ }^{B}$ Indranil Chakravorty, ${ }^{C}$ Clifford Lisk, ${ }^{D}$ Nadia Short, ${ }^{E}$ Jo Szram, ${ }^{A}$ \\ Catherine Bryant, ${ }^{\mathrm{A}}$ Karen Le Ball, ${ }^{\mathrm{A}}$ Andrew Deaner ${ }^{\mathrm{A}}$ and Jonathan Birns ${ }^{\mathrm{A}}$
}

\begin{abstract}
Aims
To deliver medical rotations across London based on the achievement of 'Capabilities in practice' set out in the Internal Medicine Training (IMT) curriculum, thereby equipping future physicians with necessary general medical skills.
\end{abstract}

\section{Methods}

Under the direction of Health Education England's Head of School of Medicine for London, a project group was convened in 2016, comprising educationalists, operational managers and physicians involved in the delivery of the core medical training programme in London for over 550 core medical trainees (CMTs). A review of existing rotations was carried out by regional training programme directors (TPDs) and School of Medicine staff to evaluate which rotations met new IMT curriculum criteria, including exposure to acute medicine, geriatrics, outpatients and critical care medicine. Two workshops were held in 2017 involving major stakeholders, including directors of medical education and the Academy of Anaesthesia, to facilitate discussion of rotational alterations and compulsory requirements of the IMT curriculum. Subsequently, regional TPDs, with support from local trainers and trainee representatives, created new rotations to meet IMT curricular requirement.

\section{Results}

Eighty per cent of core medical training rotations did not conform to IMT curriculum requirements, particularly regarding critical care exposure. One-hundred and thirty new rotations for CMTs were created, each rotation due to commence in August 2018, providing training for between four and six trainees, within north-central, north-east, north-west, south-east or south-west sectors of London. In this way, the last cohort of CMTs recruited for August 2018 was planned to rotate through an 'IMT-ready' programme ahead of

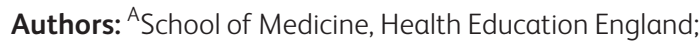
B Barts Health NHS Trust; 'St George's University Hospitals NHS

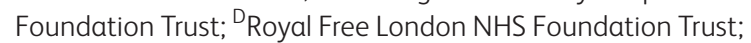
EGuy's and St Thomas' NHS Foundation Trust, London, UK the first IMT intake in August 2019. Four of the five sectors mapped 24 months of training to satisfy new curriculum requirements and one sector mapped only the first 12 months of training (ie the IMT1 year alone) with work continuing to ready the second year rotations (IMT2) in line with the other sectors. These processes required extensive local negotiations along with decommissioning and recommissioning of some posts within the same funding envelope.

\section{Conclusion}

The successful mapping of the IMT curriculum to medical trainee rotations in London demonstrated that major curriculum changes are possible at scale, with regional and local cooperation. The opportunity for any issues of concern within the new rotations to be flagged up in advance of IMT recruitment in 2019 are seen as an advantage, as well as potentially reduced burden of work for local trainers and TPDs in the lead-up to 2019 national recruitment.

\section{Conflict of interest statement}

None declared. 